Musées, Patrimoine et Culture scientifiques et techniques

147 | 2013

mai - juin 2013

\title{
Présentation de quelques documents pour l'exportation de spécimens paléontologiques depuis la Fédération de Russie
}

\section{Gilles Pacaud}

\section{OpenEdition}

\section{Journals}

Édition électronique

URL : http://journals.openedition.org/ocim/1231

DOI : 10.4000/ocim.1231

ISSN : 2108-646X

Éditeur

OCIM

Édition imprimée

Date de publication : 1 mai 2013

Pagination : 18-19

ISSN : 0994-1908

\section{Référence électronique}

Gilles Pacaud, «Présentation de quelques documents pour l'exportation de spécimens

paléontologiques depuis la Fédération de Russie », La Lettre de I'OCIM [En ligne], 147 | 2013, mis en ligne le 01 mai 2015, consulté le 01 mai 2019. URL : http://journals.openedition.org/ocim/1231 ; DOI 10.4000/ocim. 1231

Ce document a été généré automatiquement le 1 mai 2019.

Tous droits réservés 


\title{
Présentation de quelques documents pour l'exportation de spécimens paléontologiques depuis la Fédération de Russie
}

\author{
Gilles Pacaud
}

La convention de l'UNESCO concernant les mesures à prendre pour interdire et empêcher l'importation, l'exportation et le transfert de propriété illicite de biens culturels a été signée par la Fédération de Russie le 28 avril 1988 et par la France le 7 janvier 1997. Si la compréhension du texte ne pose pas de problème pour le personnel des muséums, son application concrète est plus délicate. Les modalités douanières sont internationales et destinées essentielle- ment à la perception des taxes. Ces documents douaniers sont donc indispensables, mais pas suffisants. Pour le montrer, je donnerai comme exemple des acquisitions paléontologiques venant de la Fédération de Russie. Cependant, afin de ne 
pas donner des idées à des vendeurs peu scrupuleux, je ne ferai pas de commentaires sur les points particuliers qu'il convient de vérifier et de croiser.
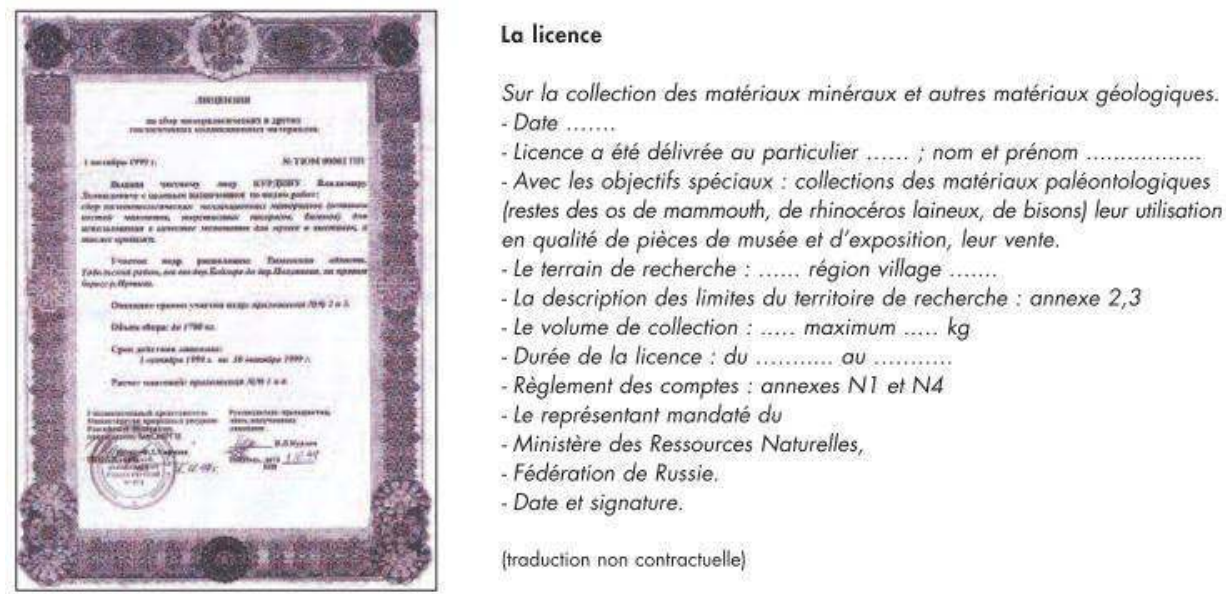

Les informations sont à croiser avec celles du certificat. Souvent, le vendeur qui fait l'exportation n'est pas le collecteur. Il est souhaitable d'avoir une attestation ou une copie de la transaction entre le récolteur et l'exportateur s'ils sont différents. À noter que la collecte est limitée dans le temps et dans l'espace. Elle est spécifique d'un groupe paléontologique.

(c) G.Pacaud

Les documents douaniers devraient être fournis et faire l'objet d'un examen critique lors d'une acquisition même dans une salle des ventes. Malheureusement, cela est rarement possible, à ma connaissance cela a été fait pour une seule vente en France, pourtant c'est une pratique commune pour les ventes en entomologie, ornithologie... Il n'y a donc rien d'impossible.
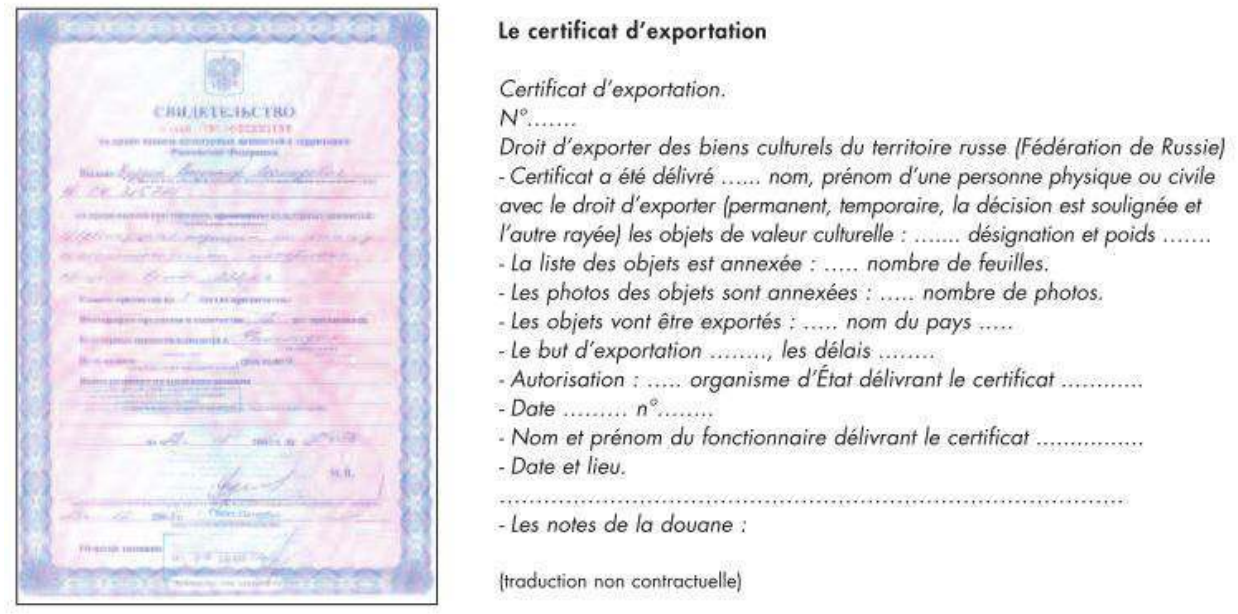

Dans cet exemple, il s'agit d'une exportation permanente de 12 objets de la liste de matériaux paléontologiques, le poids est de $232 \mathrm{~kg}$. II y a 12 photographies.

(c) G.Pacaud

3 Seul l'exemple de la Russie est donné ici. Si l'esprit de la convention est respecté par les signataires son application concrète varie d'un pays à l'autre, mais aussi dans le temps. Ainsi, avant janvier 2011, le commerce de fossiles était possible en Chine, mais l'exportation interdite, donc une situation simple à appliquer. Maintenant, les fossiles chinois sont classés au point de vue vente en 3 catégories : interdiction ou sous condition jusqu'à la vente libre avec ou sans exportation. 
Annexe au certificat d'exportation : liste des spécimens à exporter (reproduction partielle)

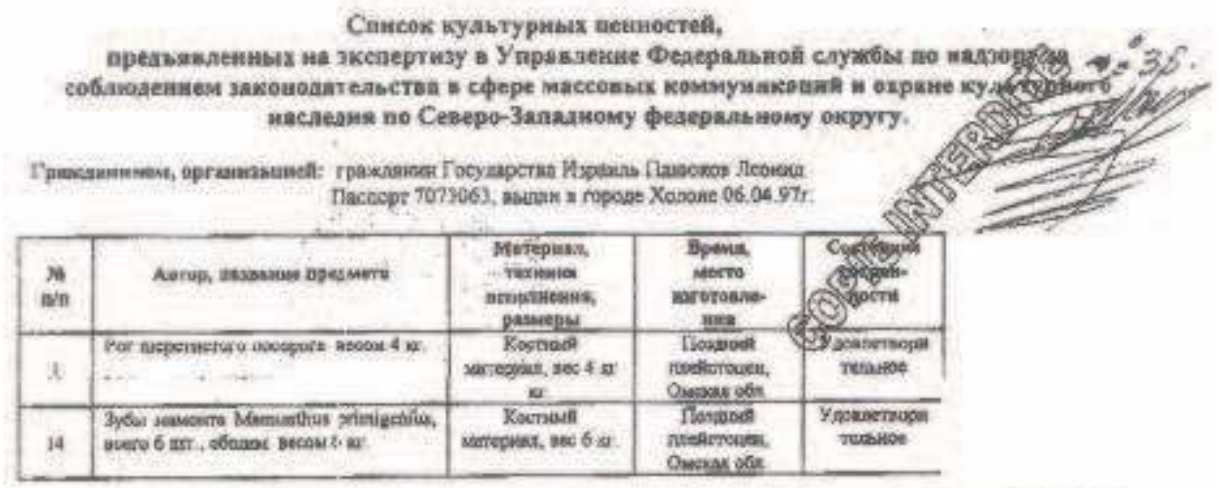

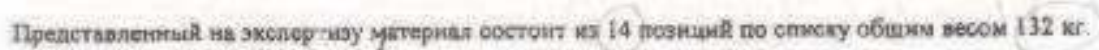

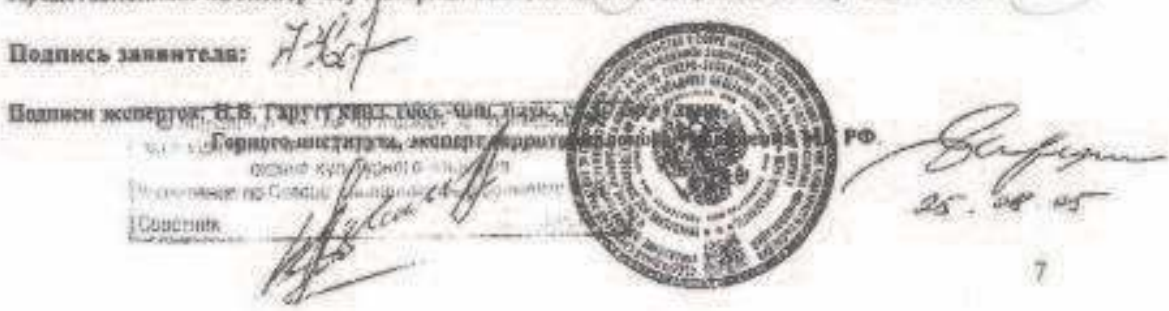

(c) G.Pacaud

Annexe au certificat d'exportation (certificat vétérinaire) : cedocument est important pour certains spécimens (le bébé mammouth « Kroma » exposé en France était contaminé par l'anthrax).

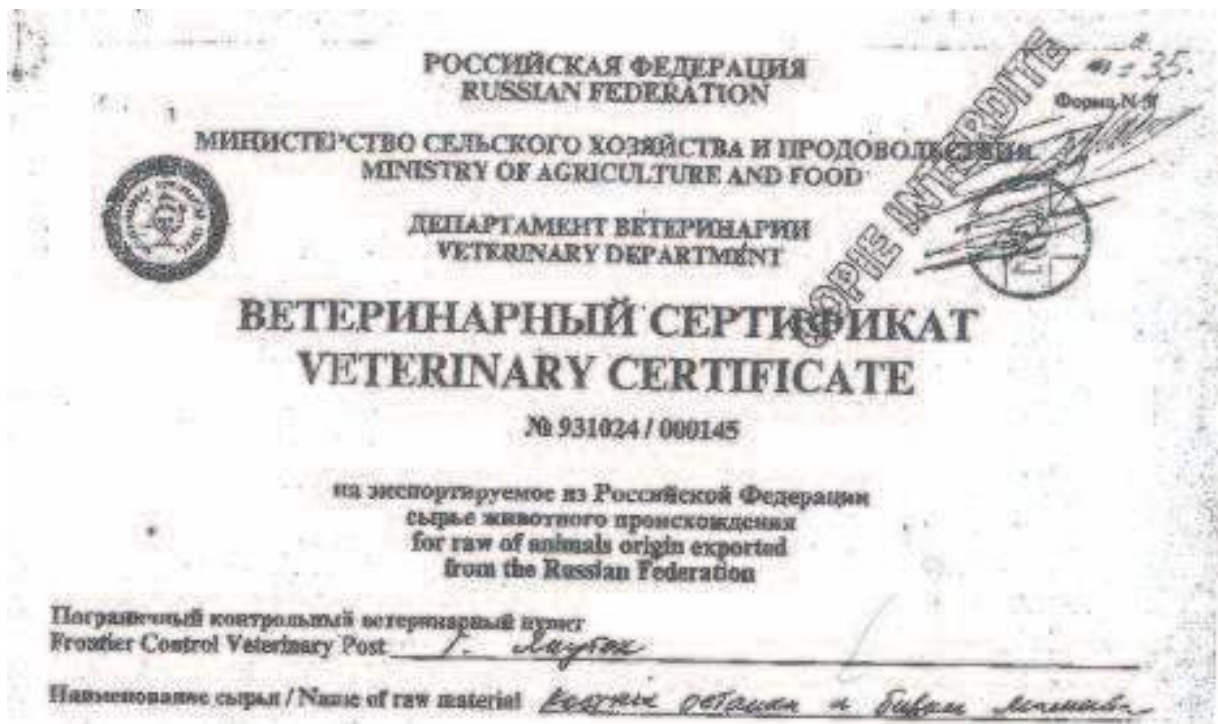

Ce certificat est indépendant du document d'accompagnement français indiquant que l'importateur ne destine pas ces produits à l'alimentation humaine ou animale (reproduction partielle).

(c) G.Pacaud

Il est à noter que les musées doivent redoubler de vigilance sur l'examen des documents d'accompagne- ment pour certaines sorties de spécimens paléontologiques du territoire de la Fédération de Russie, notamment en ce qui concerne les sorties par SaintPétersbourg et Blagovechtchensk ou via la Finlande, l'Estonie... 
INDEX

Mots-clés : collections, paléontologie

AUTEUR

GILLES PACAUD

Conservateur en chef honoraire 